\title{
Three-in-one: case of multiple primary malignancies concurrently in a 70 year-old female
}

\begin{abstract}
Multiple primary malignancies (MPM) are an increasing trend seen in oncology due to factors of increased survival time of patients, environmental exposures and hereditary risk factors. The increase of diagnoses is also seen with more screenings as well as longer life expectancies. We present a 70 year old female who initially presented with trouble swallowing. It was revealed she had squamous cell carcinoma (SCC) of her epiglottis. PET scan work up revealed uptake in her left breast. Bilateral mammogram, ultrasound and biopsy revealed her left breast had invasive ductal carcinoma (IDC) and her right had invasive lobular carcinoma (ILC). With her smoking history and strong family history of cancer, she was highly susceptible to developing multiple primary malignancies She did not continue routine health screenings including mammogram and low dose CT chest which may have prevented or discovered her malignancies at an early stage. This is an emphasis on the importance of routine health surveillance and screening especially in the primary care setting.
\end{abstract}

Volume I2 Issue 3 - 202 I

\author{
Joseph R Malhis,' Chintav Shah,' S Blake \\ O'Brien,' Rikhav Vasanwala,' Simeon \\ Jaggernauth ${ }^{2}$ \\ IInternal Medicine Resident PGY-2, University of Arkansas for \\ Medical Sciences Northwest Regional Campus, USA \\ ${ }^{2}$ Landmark Oncology Center, USA
}

Correspondence: Joseph R Malhis, DO, MA, Internal Medicine Resident PGY-2, University of Arkansas for Medical Sciences Northwest Regional Campus, Fayetteville, AR, USA, Email ymalhis@gmail.com

Received: April 27, 202I | Published: May 12, 202 |

Keywords: multiple primary malignancies, squamous cell carcinoma, invasive ductal carcinoma of the breast, invasive lobular carcinoma of the breast

\section{Background}

Multiple primary malignancies are seen when there are distinct histological and sites of origin for these malignancies to arise $\mathrm{e}^{1,2}$ and that metastasis is ruled out. ${ }^{3}$ And this is not a recent phenomenon with 3000 cases noted in 1921 finding "multiple growths". ${ }^{2}$ Some manifestations are synchronous indicating all are diagnosed within a 6-month period or metachronous meaning greater than 6 month time. There is an increased trend with patients having MPM due to increased life expectancy; increased survival of cancer patients; hereditary, immune and environmental risk factors and increased diagnosing tools. ${ }^{1-3}$ However, they are still rare with a 1.29 -fold risk of developing a new malignancy compared to one who never has. ${ }^{3}$ Between genders, there is a higher prevalence among women with most commonly seen with breast, then cervix with age ranging from $43-68 .{ }^{1}$ A study has Coyte et al followed 8,386 women diagnosed with breast cancer with 79 developing another primary breast cancer within 5 years based on the SEER guideline. ${ }^{2}$ The incidence according to Kim et al was $4.1 \%$ $16.4 \%$ for having multiple primaries with recurrence between 5 to 8 years. $^{2}$

Second primary malignancies are seen with head and neck squamous cell carcinomas and it is the second leading cause of death, seen in one-quarter to one-third of deaths. ${ }^{4}$ Based on a study with 99,000 patients, 10,826 patients had second primary cancer and based on that, the 20-year cumulative risk of a second cancer was 36 percent, and the standardized incidence ratio (SIR) compared with the general population was $1.86 .{ }^{4}$ The most common second primary cancer was in the lung with 20- year cumulative risk 13 percent, SIR 3.3. ${ }^{4}$ In addition, there is an increased risk of developing esophageal cancer as a second primary. ${ }^{4}$

Supraglottic laryngeal squamous cell carcinomas are the most common form of head and neck cancers, especially in those who consume tobacco and alcohol. HPV-positive patients are commonly seen with laryngeal carcinoma, predominantly higher in the supraglottal region with positivity of $60.6 \%$ compared to other laryngeal subregions. ${ }^{1}$ Among the supraglottic regions, the epiglottis has a $14.8 \%$ rate of expression. ${ }^{5}$ There is also a higher percentage of p16 expression at $86.6 \%$ of $\mathrm{HPV}+$ patients compared to $2.9 \%$ for $\mathrm{p} 18$ expression. $^{5}$

Breast cancer has 20 different histologic types with invasive ductal carcinoma (IDC) being the most common at $80 \%$ prevalence and invasive lobular carcinoma at 5-15\%. ${ }^{2}$ ILC tends to be more bilateral, multifocal, larger tumors, high ER and PR expression and lower HER expression compared to IDC. ${ }^{6}$ There has been an intermediate classification with both of these types referred to as IDC-L. WHO categorizes it as "having an ILC pattern in at least $50 \%$ of the tumor and an IDC pattern in between 10\% and 49\%". ${ }^{6}$ Breast Cancer specific survival curve indicates that those with IDC-L fare better than the IDC or ILC group. However, prognosis is poor for this intermediate for ER positive or negative group compared to IDC. ${ }^{6}$

\section{Case report}

This is a pleasant 70-year-old female patient with a history of hypertension and GERD and is a former smoker with a 40 pack year history presented with a chief complaint of choking when eating. She had an upper endoscopy performed and a large exophytic mass was found on the anterior surface of the epiglottis partially obstructing her airway. She was referred to otolaryngology to have a biopsy done and it confirmed p 16 positive squamous cell carcinoma in $25 \%$ tumor cells present. Following biopsy, CT scan of neck found 2.4 x 3.4 x $2.6 \mathrm{~cm}$ enhancing lesion in the epiglottis extending into the base of the tongue, consistent with a primary neoplasm. PET scan same day indicated FDG avid mass in base of epiglottis with SUV of 18.6 as suspected with right level II lymph node SUV 3.1 and left side lymph SUV of 2.7. In addition, a superior aspect of the left breast found SUV 4.4 , concerning primary malignancy. She was referred to oncology and her malignancy was staged at $\mathrm{T} 3 \mathrm{~s} \mathrm{~N} 0 / 2$.

With further discussion upon visit, it was revealed that she stopped doing mammograms 10 years prior and it was indicated her mother has a history of uterine and lung cancer and her father had esophageal cancer. 
Bilateral diagnostic mammogram and target ultrasound of her breast status post tracheostomy PEG and port placement for her SCC of her epiglottis one month later found $16 \times 12 \times 11 \mathrm{~mm}$ mass at 10 o'clock position of right breast and $3 \times 6 \mathrm{~mm}$ mass at 10 o'clock position representing cyst cluster. $10 \times 8 \times 8 \mathrm{~mm}$ lymph node seen in

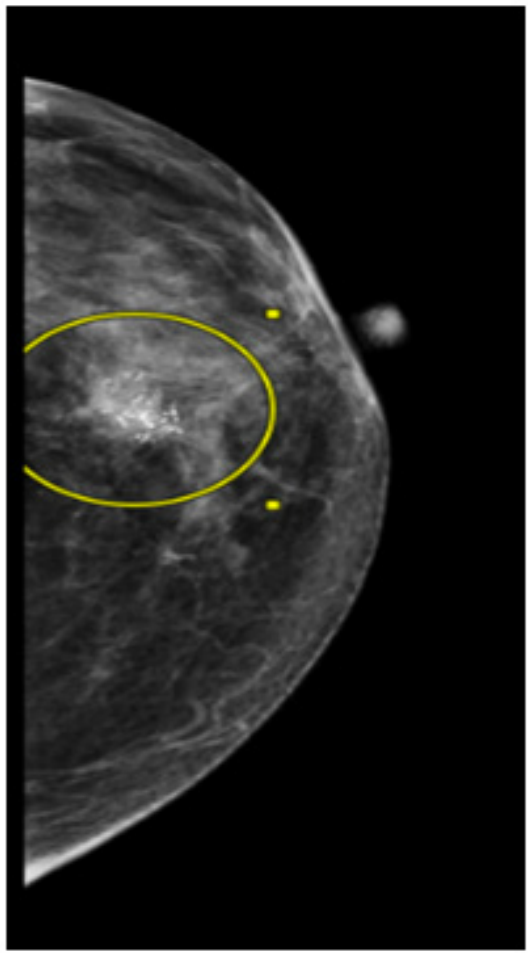

Left breast Mammogram Craniocaudal view

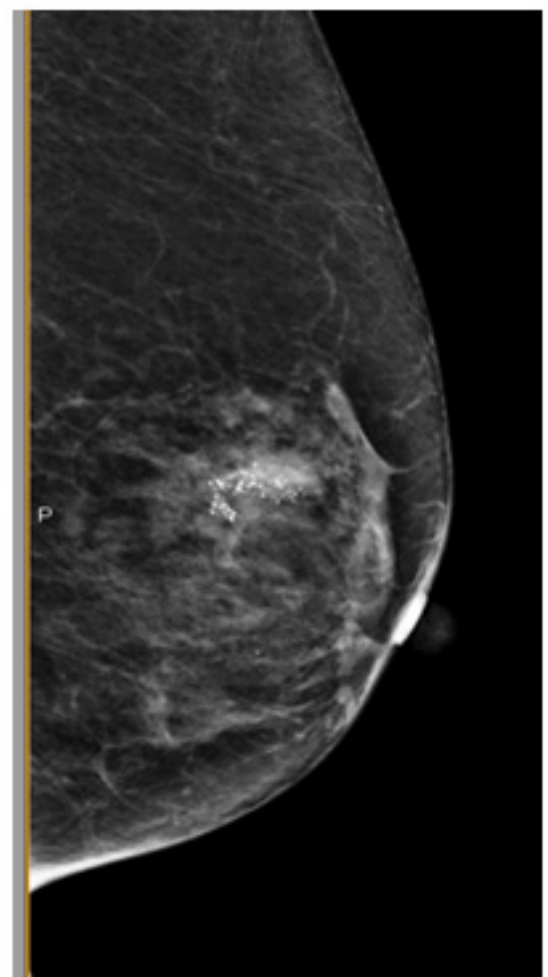

Left Breast Mammogram lateromedial oblique view the right axilla with no fatty hilum. An $18 \times 11 \times 15 \mathrm{~mm}$ mass at the 12 o'clock position of the left breast and $6 \times 3 \times 5 \mathrm{~mm}$ mass in the 5 o'clock position of the left breast representing a cyst cluster. Her BI-RADS was category 5 . Radiation therapy began the next day for her SCC.

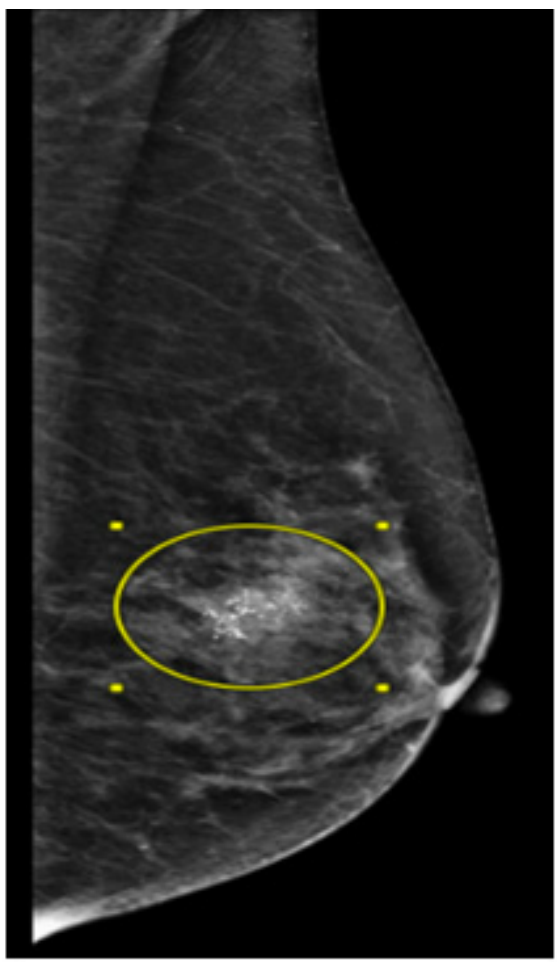

Left Breast Mammogram Lateromedial view

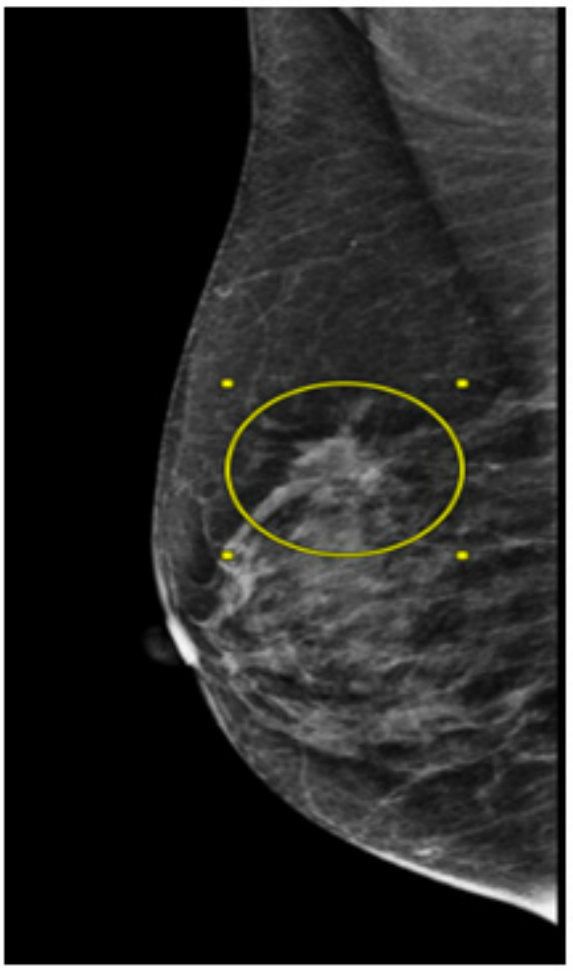

Right Breast Mammogram Mediolateral Oblique view 


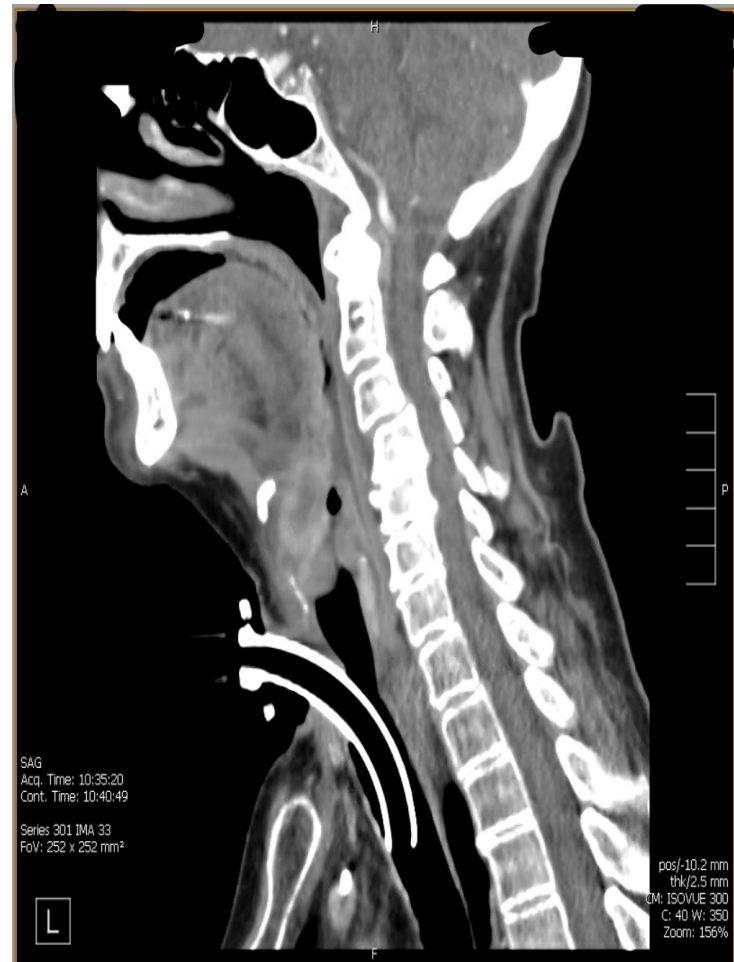

Lateral left CT view of her neck

Three days later, she had a biopsy on both breasts with her right breast showing grade 2 infiltrating lobular carcinoma in the 10 o'clock position with focal lobular carcinoma in situ and lymphovascular involvement. The breast was ER 96\%, PR 0\% Ki-67 1\% HER-2/neu $1+$ negative. Right axillary lymph node was positive for metastatic lobular carcinoma. Left breast found poorly differentiated grade 3 infiltrating ductal carcinoma with lymphovascular invasion. Biopsy was ER 96\%, PR 29\%, Ki-67 35\% HER-2/neu 0.

Three days later, she began her first cycle of Cisplatin for her SCC along with her radiation. She also began neoadjuvant endocrine therapy with Arimidex. She will have breast resection performed after completing chemotherapy. She completed radiation therapy with 5040 cGy in 30 fractions over 42 days. She continues to follow oncology.

\section{Discussion}

The cases of MPMs have been increasing due to regular screenings, increased risk factors and family history. This patient presented with 3 primary malignancies of ILC, IDC and SCC. With her smoking history, she was at an increased risk of developing SCC in the laryngeal region, especially HPV induced with $\mathrm{p} 16$. $^{7}$ She has a strong family history of cancer with her mother having lung and uterine cancer and her father with esophageal CA. In addition, she did not have a mammogram done in 10 years prior to her most recent mammogram. What we do not have on record is why she had not received the low dose CT due to her smoking history and family history of cancer. This may have been an early surveillance screening for her that may have prevented her current diagnosis of MPM. With increased vigilance of routine health screenings in primary care, her diagnosis of triple malignancies may have been prevented. The incidence of developing MPM varies from $0.73 \%$ to $11.7 \%$. After one is diagnosed with the first, there is $10 \%$ chance of developing a second tumor compared to the general population. ${ }^{8}$ With her family history, she is possibly genetically predisposed. Her mother had uterine cancer and the incidence of sporadic uterine cancer with breast cancer increases and she may also need to have BRCA screening. ${ }^{8}$ Esophageal cancer also has its inherent risk of developing another primary malignancy. Between squamous cell and adenocarcinoma, there is increased risk with SCC, especially in the oral cavity, pharynx, and larynx. Some of which can be to breast cancer, but type has not been specified. ${ }^{9}$ So, although she has all the risk factors and with regular health screening, she would not necessarily be in this current predicament. However, there are many other factors that include her socioeconomic and mental background as well as her views on her own health. It is prudent to understand the perspective patients have on his or her own health and to not presume she was not informed. Further exploration into why the patient did not wish to continue routine screenings and if she was aware of the risks involved if she were to defer treatment is indeed a lesson to learn for future practitioners.

\section{Acknowledgments}

None.

\section{Conflicts of interest}

The authors declare there is no conflict of interests regarding the publication of this paper.

\section{References}

1. Jena A, Patnayak R, Lakshmi AY, et al. Multiple primary cancers: An enigma. South Asian J Cancer. 2016;5(1):29-32.

2. Vogt A, Schmid S, Heinimann $\mathrm{K}$, et al. Multiple primary tumours: challenges and approaches, a review. ESMO Open. 2017;2(2):e000172.

3. Sakellakis M, Peroukides S, Iconomou G, et al. Multiple primary malignancies: a report of two cases. Chin J Cancer Res. 2014;26(2):215218.

4. Kang S, Theodoros NT, Marshall RP, et al. Second Primary Malignancies in Patients with Head and Neck Cancer. Uptodate.org; 2018.

5. Ding W, Liu T, Liang J, et al. Supraglottic squamous cell carcinomas have distinctive clinical features and prognosis based on subregion. PLoS One. 2017;12(11):e0188322.

6. Xiao Y, Ma D, Ruan M, et al. Mixed invasive ductal and lobular carcinoma has distinct clinical features and predicts worse prognosis when stratified by estrogen receptor status. Sci Rep. 2017;7(1):10380.

7. Haddad RI, Bruce EB, David MB, et al. HPV-associated head and neck cancer. Uptodate.org; 2019.

8. De Luca A, Frusone F, Vergine M, et al. Breast Cancer and Multiple Primary Malignant Tumors: Case Report and Review of the Literature. In Vivo. 2019;33(4):1313-1324.

9. Chen D, Fan N, Mo J, et al. Multiple primary malignancies for squamous cell carcinoma and adenocarcinoma of the esophagus. J Thorac Dis. 2019;11(8):3292-3301. 